\title{
Increase in gastroesophageal reflux disease symptoms and erosive esophagitis 1 year after laparoscopic sleeve gastrectomy among obese adults
}

\author{
Chi-Ming Tai $\cdot$ Chih-Kun Huang
}

Received: 30 April 2013/Accepted: 10 May 2013/Published online: 25 May 2013

(C) Springer Science+Business Media New York 2013

We thank Elazary et al. [1] for their interest in our article and greatly appreciate their comments.

Although the effect of laparoscopic sleeve gastrectomy (LSG) on gastroesophageal reflux disease (GERD) remains controversial, several studies have shown an increase in the prevalence of GERD after LSG. How to reduce postoperative GERD following LSG is an important question for consideration. Hiatal hernia is an important risk factor for developing GERD and its repair has been shown to decrease GERD symptoms after LSG [2,3]. Our study revealed that the occurrence of erosive esophagitis after LSG is related to the presence of hiatal hernia after the operation. We agree that modification of the surgical technique may decrease the occurrence of postoperative hiatal hernia and thereby decrease the prevalence of GERD. All our patients underwent preoperative endoscopy and if a hiatal hernia was identified, crural repair was performed. The definition of hiatal hernia used in our study was the presence of a diaphragmatic indentation that was at least $2 \mathrm{~cm}$ distal to the $\mathrm{Z}$-line and the proximal margins of the gastric mucosal

C.-M. Tai $(\bowtie) \cdot$ C.-K. Huang

Bariatric and Metabolic International Surgery Center,

E-Da Hospital, Kaohsiung, Taiwan

e-mail: chimingtai@gmail.com

C.-M. Tai

Institute of Clinical Medicine, College of Medicine, Kaohsiung

Medical University, Kaohsiung, Taiwan

\section{C.-M. Tai}

Department of Internal Medicine, E-Da Hospital/I-Shou

University, Kaohsiung, Taiwan

\section{C.-K. Huang}

Department of Surgery, E-Da Hospital/I-Shou University,

Kaohsiung, Taiwan folds. However, in using this definition, there was a probability of missing small hiatal hernias. In addition to hiatal hernia repair, we also make technical modifications in our LSG procedure to decrease stricture formation at the incisura angularis. We believe that this modification may also help reduce the incidence of postoperative GERD [4].

GERD symptoms are often used for the assessment of GERD following LSG. However, it is worth noting that GERD symptoms are not consistent with the presence of erosive esophagitis. As demonstrated in our study, $40.1 \%$ of patients who did not have postoperative GERD symptoms still had postoperative erosive esophagitis on endoscopy. Therefore, we propose that postoperative follow-up endoscopy is necessary to identify the true prevalence of postoperative GERD. Endoscopy could also show the anatomical changes in the gastric tube following modification in the surgical technique.

Disclosures C.-M. Tai and C.-K. Huang have no conflicts of interest or financial ties to disclose.

\section{References}

1. Elazary R, Phillips EH, Cunneen S, Burch MA (2013) Comments on "Increase in gastroesophageal reflux disease symptoms and erosive esophagitis 1 year after laparoscopic sleeve gastrectomy among obese adults" (doi:10.1007/s00464-012-2593-9). Surg Endosc. doi:10.1007/s00464-013-2974-8

2. Daes J, Jimenez ME, Said N, Daza JC, Dennis R (2012) Laparoscopic sleeve gastrectomy: symptoms of gastroesophageal reflux can be reduced by changes in surgical technique. Obes Surg 22:1874-1879

3. Soricelli E, Iossa A, Casella G, Abbatini F, Calì B, Basso N (2012) Sleeve gastrectomy and crural repair in obese patients with gastroesophageal reflux disease and/or hiatal hernia. Surg Obes Relat Dis (in press)

4. Zachariah SK, Chang PC, Ooi AS, Hsin MC, Kin Wat JY, Huang CK (2013) Laparoscopic sleeve gastrectomy for morbid obesity: 5 years experience from an Asian Center of Excellence. Obes Surg (in press) 